\title{
A HARDY-TYPE INEQUALITY AND SOME SPECTRAL CHARACTERIZATIONS FOR THE DIRAC-COULOMB OPERATOR
}

\author{
BIAGIO CASSANO, FABIO PIZZICHILLO AND LUIS VEGA
}

\begin{abstract}
We prove a sharp Hardy-type inequality for the Dirac operator. We exploit this inequality to obtain spectral properties of the Dirac operator perturbed with Hermitian matrixvalued potentials $\mathbf{V}$ of Coulomb type: we characterise its eigenvalues in terms of the BirmanSchwinger principle and we bound its discrete spectrum from below, showing that the groundstate energy is reached if and only if $\mathbf{V}$ verifies some rigidity conditions. In the particular case of an electrostatic potential, these imply that $\mathbf{V}$ is the Coulomb potential.
\end{abstract}

\section{IntRoduction AND MAIN RESUlts}

Firstly formulated in [14], the Hardy inequality can be stated as follows: for $d \geq 3$, the following holds

$$
\frac{(d-2)^{2}}{4} \int_{\mathbb{R}^{d}} \frac{|f|^{2}}{|x|^{2}} d x \leq \int_{\mathbb{R}^{d}}|\nabla f|^{2} d x, \quad \text { for } f \in C_{c}^{\infty}\left(\mathbb{R}^{d}\right) .
$$

This inequality is sharp, in the sense that the constant in the left hand side can not be increased, and there exists a sequence of approximate attainers. We refer to [20] for a historical review on the topic. The Hardy inequality is an uncertainty principle: it states that a function cannot be concentrated around one point (the origin) unless its momentum is big, and vice-versa if its momentum is small then the function has to be spread in the space. More in general, the Hardy inequality answers to the fundamental need in the mathematics of comparing $L^{2}$-weighted norms of a function with the norm of its derivative. In this paper, we are interested in Hardytype inequalities for the Dirac operator: we exploit them to show spectral properties of the Dirac operator perturbed with potentials of Coulomb type.

The free Dirac operator in $\mathbb{R}^{3}$ is defined by

$$
H_{0}:=-i \alpha \cdot \nabla+m \beta=-i \sum_{j=1}^{3} \alpha_{j} \partial_{j}+m \beta,
$$

where $m>0$,

$$
\beta:=\left(\begin{array}{cc}
\mathbb{I}_{2} & 0 \\
0 & -\mathbb{I}_{2}
\end{array}\right), \quad \alpha:=\left(\alpha_{1}, \alpha_{2}, \alpha_{3}\right), \quad \alpha_{j}:=\left(\begin{array}{cc}
0 & \sigma_{j} \\
\sigma_{j} & 0
\end{array}\right) \quad \text { for } j=1,2,3,
$$

and $\sigma_{j}$ are the Pauli matrices

$$
\sigma_{1}=\left(\begin{array}{cc}
0 & 1 \\
1 & 0
\end{array}\right), \quad \sigma_{2}=\left(\begin{array}{cc}
0 & -i \\
i & 0
\end{array}\right), \quad \sigma_{3}=\left(\begin{array}{cc}
1 & 0 \\
0 & -1
\end{array}\right) .
$$

Date: October 3, 2018.

2010 Mathematics Subject Classification. Primary 81Q10; Secondary 47N20, 35P05, 47B25.

Key words and phrases. Dirac operator, Coulomb potential, Hardy inequality, self-adjoint operator, spectral properties, ground state. 
It is well known (see [25]) that $H_{0}$ is self-adjoint on $H^{1}\left(\mathbb{R}^{3}\right)^{4}$ and essentially self-adjoint on $C_{c}^{\infty}\left(\mathbb{R}^{3}\right)^{4}$, moreover $\sigma\left(H_{0}\right)=\sigma_{\text {ess }}\left(H_{0}\right)=(-\infty,-m] \cup[m,+\infty)$.

In [16], Kato considered a general matrix-valued potential $\mathbf{V}: \mathbb{R}^{3} \rightarrow \mathbb{C}^{4 \times 4}$ such that $\mathbf{V}(x)$ is Hermitian for almost all $x \in \mathbb{R}^{3}$ and

$$
|\mathbf{V}(x)|:=\sup _{u \in \mathbb{C}^{4}} \frac{|\mathbf{V}(x) u|}{|u|} \leq \frac{a}{|x|}+b, \quad \text { for a.a. } x \in \mathbb{R}^{3},
$$

for some $a, b \in \mathbb{R}$. Exploiting the Kato-Rellich perturbation theory, he showed that if $a<1 / 2$ then $H:=H_{0}+\mathbf{V}$ is self-adjoint on $H^{1}\left(\mathbb{R}^{3}\right)^{4}$ and essentially self-adjoint on $C_{c}^{\infty}\left(\mathbb{R}^{3}\right)^{4}$ (for a proof see also [18, Theorem V 5.10]). A fundamental ingredient of his proof is the Hardy inequality (1.1) when $d=3$.

In general, when $a>1 / 2$ the operator $H$ is not essentially self-adjoint, as shown by Arai in [1], but the phenomena change when the potential $\mathbf{V}$ has some particular structure. For example, when $\mathbf{V}$ is the Coulomb potential $\mathbf{V}_{C}(x):=\nu /|x| \mathbb{I}_{4}$ and $|\nu|<\sqrt{3} / 2$, the operator $H_{0}+\mathbf{V}_{C}$ is self-adjoint on $H^{1}\left(\mathbb{R}^{3}\right)^{4}$ and essentially self-adjoint on $C_{c}^{\infty}\left(\mathbb{R}^{3}\right)^{4}$, as shown in [22, 27, 13]. These results suggest that the Hardy inequality (1.1) is not optimal for the study of the self-adjointness of perturbed Dirac operators, since it does not catch its matrix nature: convenient Hardy-type inequalities for the Dirac operator have to be considered. Indeed, in [24] Schmincke considered a Coulomb-type potential such that

$$
\mathbf{V}_{S}(x)=V_{S}(x) \mathbb{I}_{4}, \quad V_{S}: \mathbb{R}^{3} \rightarrow \mathbb{R}, \quad \sup _{x \in \mathbb{R}^{3}}|x|\left|V_{S}(x)\right|<\frac{\sqrt{3}}{2},
$$

and he showed that $H_{0}+\mathbf{V}_{S}$ is essentially self-adjoint. The basic idea in his proof is to introduce a suitable intercalary operator $T$ and to regard $\mathbf{V}_{S}-T$ as a perturbation of $H_{0}+T$. After a careful reading of his proof, one realises that he proved and used the following Hardy-type inequality:

$$
\int_{\mathbb{R}^{3}}\left|\left(-i \alpha \cdot \nabla+\frac{i \alpha \cdot \hat{x}}{2|x|}\right) \psi\right|^{2} d x \geq \int_{\mathbb{R}^{3}} \frac{|\psi|^{2}}{|x|^{2}} d x, \quad \text { for } \psi \in C_{c}^{\infty}\left(\mathbb{R}^{3}\right)^{4},
$$

being $\hat{x}:=x /|x|$. In fact, thanks to (1.2), we have

$$
\left|\left(\mathbf{V}_{S}(x)-\frac{i \alpha \cdot x}{2|x|^{2}}\right) \psi\right|^{2}=\left(\left|V_{S}(x)\right|^{2}+\frac{1}{4|x|^{2}}\right)|\psi|^{2} \leq \frac{|\psi|^{2}}{|x|} .
$$

The result of Schmincke is not an immediate application of the Kato-Rellich theory, since the operator $i \alpha \cdot \hat{x} / 2|x|$ is not symmetric: see [24] for more details.

For $|\nu|>\sqrt{3} / 2$ the operator $H_{0}+\mathbf{V}_{C}$ is not essentially self-adjoint on $C_{c}^{\infty}\left(\mathbb{R}^{3}\right)^{4}$ and infinite self-adjoint extensions can be constructed. Among all, when $|\nu|<1$ there exists a unique selfadjoint extension $H_{D}$, characterized by the fact that

$$
\mathcal{D}\left(H_{D}\right) \subset \mathcal{D}\left(r^{-1 / 2}\right)^{4} \quad \text { or equivalently } \quad \mathcal{D}\left(H_{D}\right) \subseteq H^{1 / 2}\left(\mathbb{R}^{3}\right)^{4},
$$

where

$$
\mathcal{D}\left(r^{-1 / 2}\right)^{4}:=\left\{\psi \in L^{2}\left(\mathbb{R}^{3}\right)^{4}:|x|^{-1 / 2} \psi \in L^{2}\left(\mathbb{R}^{3}\right)^{4}\right\},
$$

see $[4,12,19,21,23,28,12,11]$. Since $H_{D}$ is the unique self-adjoint extension verifying (1.3), it is called distinguished because it is the most physically meaningful extension. 
In [17] Kato constructed the distinguished self-adjoint extension in the general case that $\mathbf{V}$ is a Hermitian matrix-valued potential such that

$$
\sup _{x \in \mathbb{R}^{3}}|x||\mathbf{V}(x)|=: \nu<1 .
$$

To prove his result, Kato exploited the following 4-spinor Hardy-type inequality, firstly conjectured by Nenciu in [21]:

$$
\int_{\mathbb{R}^{3}} \frac{|\psi|^{2}}{|x|} d x \leq \int_{\mathbb{R}^{3}}|(-i \alpha \cdot \nabla+m \beta \pm i) \psi|^{2}|x| d x, \quad \text { for } \psi \in C_{c}^{\infty}\left(\mathbb{R}^{3}\right)^{4} .
$$

Finally in [2,3], by means of the Kato-Nenciu inequality (1.6), it is proved that

$$
\mathcal{D}\left(H_{D}\right)=\left\{\psi \in \mathcal{D}\left(r^{-1 / 2}\right)^{4}:\left(H_{0}+\mathbf{V}\right) \psi \in L^{2}\left(\mathbb{R}^{3}\right)^{4}\right\},
$$

being $\mathcal{D}\left(r^{-1 / 2}\right)^{4}$ defined in (1.4).

In [8], Dolbeault, Esteban and Séré proved the validity of a min-max formula to determine the eigenvalues in the gap of the essential spectrum of the Dirac operator perturbed with Coulomblike potentials $\mathbf{V}$ such that

$$
\mathbf{V}(x):=V(x) \mathbb{I}_{4}, \quad \lim _{|x| \rightarrow+\infty}|V(x)|=0, \quad-\frac{\nu}{|x|}-c_{1} \leq V \leq c_{2}:=\sup (V),
$$

with $\nu \in(0,1)$ and $c_{1}, c_{2} \geq 0, c_{1}+c_{2}-1<\sqrt{1-\nu^{2}}$. As a consequence of their results, they proved the following Hardy-type inequality:

$$
\int_{\mathbb{R}^{3}} \frac{|\sigma \cdot \nabla \varphi|^{2}}{a+\frac{1}{|x|}}+\int_{\mathbb{R}^{3}}\left(a-\frac{1}{|x|}\right)|\varphi|^{2} \geq 0, \quad \text { for all } a>0, \varphi \in C_{c}^{\infty}\left(\mathbb{R}^{3}\right)^{2},
$$

see also [7] for a later direct analytical proof. Thanks to this inequality, in [10], Esteban and Loss considered a general electrostatic potential $V: \mathbb{R}^{3} \rightarrow \mathbb{R}$ such that that for some constant $c(V) \in(-1,1), \Gamma:=\sup (V)<1+c(V)$ and for every $\varphi \in C_{c}^{\infty}\left(\mathbb{R}^{3}, \mathbb{C}^{2}\right)$,

$$
\int_{\mathbb{R}^{3}}\left(\frac{|\sigma \cdot \nabla \varphi|^{2}}{1+c(V)-V}+(1+c(V)+V)|\varphi|^{2}\right) d x \geq 0,
$$

and, for $\mathbf{V}:=V \mathbb{I}_{4}$, they proved that the operator $H_{0}+\mathbf{V}$ is self-adjoint on the appropriate domain. In particular, they could treat potentials such that

$$
-\frac{\nu}{|x|} \leq V(x)<1+\sqrt{1-\nu^{2}}, \quad \text { with } \nu \in(0,1],
$$

obtaining the distinguished extension in the case that $\nu<1$, and giving a definition of distinguished extension in the critical case $\nu=1$. The inequality (1.9) was then used by Esteban, Lewin and Séré to study the spectrum of the Dirac operator perturbed with these potentials of this kind: in [9] they provided details on the domain of the distinguished extension and they showed the validity of a min-max formula for the eigenvalues in the spectral gap. In order to give properties on the spectrum of the Dirac operator perturbed with a general Coulomb-type Hermitian matrix-valued potential, in the following theorem we prove a generalized version of (1.6). In it we use the spin angular momentum operator $\mathbf{S}$ and the orbital angular momentum $L$, whose definitions can be found in (A.2).

Theorem 1.1. Let $m>0$ and $a \in(-m, m)$. Let $\psi$ be a distribution such that

$$
\int_{\mathbb{R}^{3}}|(-i \alpha \cdot \nabla+m \beta-a) \psi|^{2}|x| d x<+\infty .
$$


Then $\psi,(1+2 \mathbf{S} \cdot L) \psi \in L^{2}\left(|x|^{-1}\right)^{4}$ and

$$
\frac{m^{2}-a^{2}}{m^{2}} \int_{\mathbb{R}^{3}} \frac{|\psi|^{2}}{|x|} d x \leq \frac{m^{2}-a^{2}}{m^{2}} \int_{\mathbb{R}^{3}} \frac{|(1+2 \mathbf{S} \cdot L) \psi|^{2}}{|x|} d x \leq \int_{\mathbb{R}^{3}}|(-i \alpha \cdot \nabla+m \beta-a) \psi|^{2}|x| d x .
$$

The inequalities are sharp, in the sense that the constants on the left hand side can not be improved. If $a \neq 0$, all the attainers of (1.13) are given by the elements of the two(complex)parameter family $\left\{\psi_{C}^{a}\right\}_{C \in \mathbb{C}^{2}}$, with

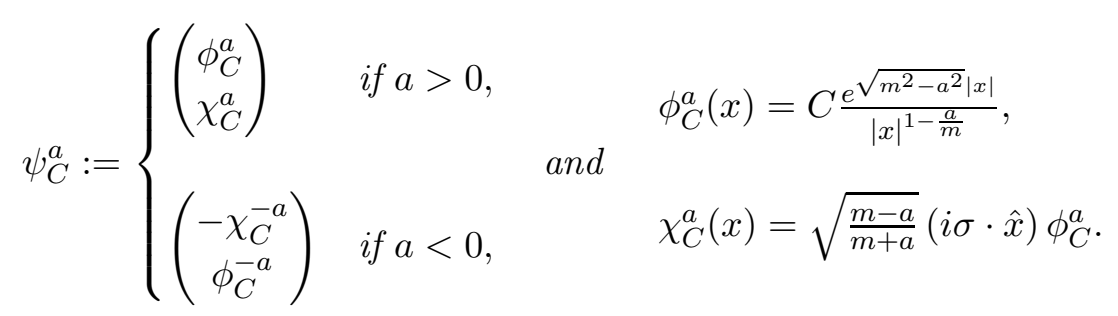

Remark 1.2. In the case that $a=0$, the inequality (1.13) is attained by the functions $\psi_{C}^{a}$ defined in (1.14), setting $a=0$, in the sense that

$$
\lim _{\epsilon \rightarrow 0} \int_{\{|x|>\epsilon\}}\left[|x|\left|(-i \alpha \cdot \nabla+m \beta) \psi_{C}^{0}\right|^{2}-\frac{\left|\psi_{C}^{0}\right|^{2}}{|x|}\right] d x=0 .
$$

In the following we exploit Theorem 1.1 to describe the discrete spectrum of the distinguished realization $H_{D}$ defined in (1.7), when (1.5) holds. We refer to [17, 2, 3] for details on its definition and properties.

From [25, Theorem 4.7] we know that

$$
\sigma_{\text {ess }}\left(H_{D}\right)=\sigma_{\text {ess }}\left(H_{0}\right)=(-\infty,-m] \cup[m,+\infty),
$$

and the discrete spectrum $\sigma_{d}\left(H_{D}\right) \subset(-m, m)$.

Thanks to Theorem 1.1, for $a \in(-m, m)$

$$
\left(H_{0}-a\right)^{-1}: L^{2}(|x|)^{4} \rightarrow L^{2}\left(|x|^{-1}\right)^{4} \quad \text { is well-defined and bounded, }
$$

and so we immediately deduce that

$$
\mathbf{u}\left(H_{0}-a\right)^{-1} \mathbf{v}: L^{2}\left(\mathbb{R}^{3}\right)^{4} \rightarrow L^{2}\left(\mathbb{R}^{3}\right)^{4} \quad \text { is well-defined and bounded, }
$$

where

$$
\mathbf{u}(x):=|x|^{1 / 2} \mathbf{V}(x) \quad \text { and } \quad \mathbf{v}(x):=|x|^{-1 / 2} \mathbb{I}_{4} .
$$

Thanks to this, in the following theorem we characterize all the eigenvalues in $(-m, m)$ of the operator $H_{D}$ in terms of a Birman-Schwinger principle.

Theorem 1.3 (Birman-Schwinger principle). Let $\mathbf{V}$ be a Hermitian matrix-valued potential that verifies (1.5), and let $\mathbf{u}, \mathbf{v}$ be defined as in (1.16). Let $H_{D}$ be the distinguished self-adjoint realization defined in (1.7), and let $a \in(-m, m)$. Then

$$
a \in \sigma_{d}\left(H_{D}\right) \Longleftrightarrow-1 \in \sigma_{d}\left(\mathbf{u}\left(H_{0}-a\right)^{-1} \mathbf{v}\right) .
$$

Moreover, the multiplicity of a as an eigenvalue of $H_{D}$ coincides with the multiplicity of -1 as an eigenvalue of $\mathbf{u}\left(H_{0}-a\right)^{-1} \mathbf{v}$. 
The discrete spectrum of the distinguished self-adjoint realization of $H_{\nu}:=H_{0}-\frac{\nu}{|x|}$ with $0<\nu<1$, is given by

$$
\sigma_{d}\left(H_{\nu}\right):=\left\{a_{1}, a_{2}, \ldots\right\}, \quad m \sqrt{1-\nu^{2}}=a_{1} \leq a_{2} \leq \cdots \leq a_{n} \leq \cdots \leq m, \quad \lim _{n \rightarrow+\infty} a_{n}=m,
$$

see [25] for more details. It is easy to check that for any $C \in \mathbb{C}^{2}$ :

$$
\left(H_{0}-\frac{\nu}{|x|}-a_{1}\right) \psi_{C}^{a_{1}}=0
$$

being $\psi_{C}^{a_{1}}$ defined in (1.14). In other words, the attainers of (1.13) are eigenvalues of the Dirac operator coupled with the Coulomb potential. Moreover, it is easy to prove that $a \in \sigma_{d}\left(H_{\nu}\right)$ if and only if $-a \in \sigma_{d}\left(H_{-\nu}\right)$. So $\sigma_{d}\left(H_{-\nu}\right)=\left\{-a_{1},-a_{2}, \ldots\right\}$, and for any $C \in \mathbb{C}^{2}$ :

$$
\left(H_{0}+\frac{\nu}{|x|}+a_{1}\right) \psi_{C}^{-a_{1}}=0
$$

In $[8,7]$, it is considered a radially symmetric electrostatic potential as in $(1.8)$, with $\nu \in(0,1)$ and $c_{1}, c_{2} \geq 0, c_{1}+c_{2}-1<\sqrt{1-\nu^{2}}$. It is proved that the discrete spectrum of the distinguished self-adjoint realization $H_{D}$ is given by

$$
\sigma_{d}\left(H_{\mathcal{D}}\right):=\left\{a_{1}, a_{2}, \ldots\right\}, \quad m \sqrt{1-\nu^{2}} \leq a_{1} \leq a_{2} \leq \cdots \leq a_{n} \leq \cdots \leq m, \quad \lim _{n \rightarrow+\infty} a_{n}=m .
$$

Finally, in [9], this was proved when (1.11) holds true.

From (1.17) and (1.20), we have that the value $m \sqrt{1-\nu^{2}}$ is the lower bound for the absolute value of the elements of discrete spectrum in presence of an electrostatic potential. Such lower bound is reached in the case of the Coulomb potential, as shown in (1.18) and (1.19). Exploiting Theorem 1.1, in the next theorem we show that this phenomenon is more general, proving that $m \sqrt{1-\nu^{2}}$ is the lower bounds for the absolute value of the elements of the discrete spectrum of $H_{D}$ for a general Hermitian matrix-valued potential $\mathbf{V}$ satisfying (1.5). Moreover we can characterize all the potentials $\mathbf{V}$ such that the values $\pm m \sqrt{1-\nu^{2}}$ are eigenvalues for $H_{D}$.

Theorem 1.4. Let $\mathbf{V}$ be a Hermitian matrix valued potential that verifies (1.5), and let $H_{D}$ be the distinguished self-adjoint realization defined in (1.7). Let $a \in \sigma_{d}\left(H_{D}\right)$, let $\mu(a)$ be its multiplicity and let $\psi \in \mathcal{D}\left(H_{D}\right)$ be an associated eigenfunction. Then, the following hold:

(i) $|a| \geq m \sqrt{1-\nu^{2}}$;

(ii) $a= \pm m \sqrt{1-\nu^{2}}$ if and only if $\psi=\psi_{C}^{a}$ for some $C \in \mathbb{C}^{2}$, where $\psi_{C}^{a}$ is defined in (1.14); in this case, $\mathbf{V} \psi_{C}^{a}=\mp \frac{\nu}{|x|} \psi_{C}^{a}$ and $\mu(a) \leq 2$;

(iii) in the case that $a= \pm m \sqrt{1-\nu^{2}}$, then $\mu(a)=2$ if and only if

$$
\mathbf{V}(x)=\left\{\begin{array}{ll}
-\frac{\nu}{|x|} \mathbb{I}_{4}+\left(\begin{array}{cc}
\mathrm{N}^{2} \sigma \cdot \hat{x} \mathbf{W}^{+}(x) \sigma \cdot \hat{x} & i \mathrm{~N} \sigma \cdot \hat{x} \mathbf{W}^{+}(x) \\
-i \mathrm{NW}^{+}(x) \sigma \cdot \hat{x} & \mathbf{W}^{+}(x)
\end{array}\right) & \text { if } a>0, \\
\frac{\nu}{|x|} \mathbb{I}_{4}+\left(\begin{array}{cc}
\mathbf{W}^{-}(x) & i \mathrm{NW}^{-}(x) \sigma \cdot \hat{x} \\
-i \mathrm{~N} \sigma \cdot \hat{x} \mathbf{W}^{-}(x) & \mathrm{N}^{2} \sigma \cdot \hat{x} \mathbf{W}^{-}(x) \sigma \cdot \hat{x}
\end{array}\right) & \text { if } a<0,
\end{array} \quad \text { for } x \neq 0,\right.
$$

where $\mathrm{N}=\sqrt{\frac{1-\sqrt{1-\nu^{2}}}{1+\sqrt{1-\nu^{2}}}}$, and $\mathbf{W}^{+}(x)$ and $\mathbf{W}^{-}(x)$ are $2 \times 2$ Hermitian matrices whose eigenvalues are respectively $\left\{\lambda_{j}^{+}(x)\right\}_{j=1,2}$ and $\left\{\lambda_{j}^{-}(x)\right\}_{j=1,2}$, and they verify

$$
-\frac{\nu}{|x|}\left(1+\sqrt{1-\nu^{2}}\right) \leq \lambda_{j}^{-}(x) \leq 0 \leq \lambda_{j}^{+}(x) \leq \frac{\nu}{|x|}\left(1+\sqrt{1-\nu^{2}}\right), \quad \text { for } j=1,2 .
$$


Remark 1.5. From (i) we directly have that $0 \notin \sigma_{d}\left(H_{D}\right)$.

An immediate consequence of Theorem 1.4 is the following corollary.

Corollary 1.6. Let $\mathbf{V}(x)=V(x) \mathbb{I}_{4}$, with $V: \mathbb{R}^{3} \rightarrow \mathbb{R}$, and such that (1.5) holds. Let $a \in \sigma_{d}\left(H_{D}\right)$ and assume that $a= \pm m \sqrt{1-\nu^{2}}$. Then $V(x)=\mp \frac{\nu}{|x|}$.

In this paper we are considering $\nu<1$ in (1.5), since in the critical case, namely when $\nu=1$ in (1.5), a definition of distinguished extension is not available for a general Hermitian matrixvalued potential. Indeed, in the particular case of the Coulomb potential $\mathbf{V}_{C}(x)=\nu /|x| \mathbb{I}_{4}$, when $|\nu| \geq 1$ many self-adjoint extensions can be built: for $|\nu|>1$ none appears to be distinguished in some suitable sense, see [15, 26, 29]. For electrostatic potentials such that (1.5) holds with $\nu=1$, a definition of distinguished extension is implied by the results of [10], and in [9] it is shown that this extension is the physically relevant one since it is the limit in the norm resolvent sense of potentials where the singularity has been removed with a cut-off. For the operator $H_{0}+\mathbf{V}_{\text {rad }}$, where

$$
\mathbf{V}_{\text {rad }}(x):=\frac{1}{|x|}\left(\nu \mathbb{I}_{4}+\mu \beta-i \lambda \alpha \cdot \hat{x} \beta\right), \quad \text { for } \nu, \mu, \lambda \in \mathbb{R},
$$

a complete description of all the self-adjoint extensions is given in [6, 5]. Under some conditions on the size of the constants $\nu, \mu, \lambda$, a distinguished extension is selected by means of a Hardy-type inequality and a quadratic form approach. Nevertheless, in the particular case of the anomalous magnetic potential $\mathbf{V}(x)= \pm i \alpha \cdot \hat{x} \beta|x|^{-1}$ such criteria do not appear to be strong enough to select any extension, see [6, Remark 1.10] and [5, Remark 1.6].

This paper is organised as follows: in Section 2 we prove Theorem 1.1, in Section 3 we prove Theorem 1.3 and we prove Theorem 1.4 in Section 4; finally, in Appendix A we recall the partial wave decomposition and related properties.

Acknowledgements. This research is supported by ERCEA Advanced Grant 2014669689 HADE, by the MINECO project MTM2014-53850-P, by Basque Government project IT-641-13 and also by the Basque Government through the BERC 2014-2017 program and by Spanish Ministry of Economy and Competitiveness MINECO: BCAM Severo Ochoa excellence accreditation SEV-2013-0323. The first author also acknowledges the Istituto Italiano di Alta Matematica "F. Severi" and the Czech Science Foundation (GAČR) within the project 17-01706S.

\section{Hardy-type inequalities for the Dirac Operator}

We use the following abstract result.

Lemma 2.1. Let $\mathcal{S}, \mathcal{A}$ be respectively a symmetric and an anti-symmetric operator on a complex Hilbert space $\mathcal{H}$. Then the following holds:

$$
2 \operatorname{Re}\langle\mathcal{A} u, \mathcal{S} u\rangle_{\mathcal{H}}=\langle[\mathcal{S}, \mathcal{A}] u, u\rangle_{\mathcal{H}},
$$

where $[\mathcal{S}, \mathcal{A}]:=\mathcal{S} \mathcal{A}-\mathcal{A S}$ is the commutator of the operators $\mathcal{S}$ and $\mathcal{A}$.

Proof. The proof is a simple computation:

$$
2 \operatorname{Re}\langle\mathcal{A} u, \mathcal{S} u\rangle_{\mathcal{H}}=\langle\mathcal{A} u, \mathcal{S} u\rangle_{\mathcal{H}}+\overline{\langle\mathcal{A} u, \mathcal{S} u\rangle_{\mathcal{H}}}=\langle\mathcal{S} \mathcal{A} u, u\rangle_{\mathcal{H}}-\langle\mathcal{A S} u, u\rangle_{\mathcal{H}} .
$$


Proof of Theorem 1.1. Let us firstly assume that $\psi \in \mathcal{S}\left(\mathbb{R}^{3}\right)^{4}$. The proof descends immediately from the explicit computation of the following square:

$$
\begin{aligned}
0 & \leq \int_{\mathbb{R}^{3}}|x|\left|(-i \alpha \cdot \nabla+m \beta-a) \psi-i \alpha \cdot \hat{x}\left(1-\frac{a}{m} \beta\right)(1+2 \mathbf{S} \cdot L) \frac{\psi}{|x|}\right|^{2} d x \\
& =\int_{\mathbb{R}^{3}}|x||(-i \alpha \cdot \nabla+m \beta-a) \psi|^{2} d x-\frac{m^{2}-a^{2}}{m^{2}} \int_{\mathbb{R}^{3}} \frac{|(1+2 \mathbf{S} \cdot L) \psi|^{2}}{|x|} d x .
\end{aligned}
$$

Thanks to the fact that

$$
\begin{aligned}
& \int_{\mathbb{R}^{3}}|x|\left|(-i \alpha \cdot \nabla+m \beta-a) \psi-i \alpha \cdot \hat{x}\left(1-\frac{a}{m} \beta\right)(1+2 \mathbf{S} \cdot L) \frac{\psi}{|x|}\right|^{2} d x \\
& =\int_{\mathbb{R}^{3}}|x||(-i \alpha \cdot \nabla+m \beta-a) \psi|^{2} d x+\int_{\mathbb{R}^{3}}|x|\left|\left(1-\frac{a}{m} \beta\right)(1+2 \mathbf{S} \cdot L) \frac{\psi}{|x|}\right|^{2} d x \\
& -2 \operatorname{Re} \int_{\mathbb{R}^{3}}(-i \alpha \cdot \nabla+m \beta-a) \psi \cdot \overline{i \alpha \cdot \hat{x}\left(1-\frac{a}{m} \beta\right)(1+2 \mathbf{S} \cdot L) \psi} d x,
\end{aligned}
$$

to prove (2.1) it is enough to prove that

$$
\begin{aligned}
& -2 \operatorname{Re} \int_{\mathbb{R}^{3}}(-i \alpha \cdot \nabla+m \beta-a) \psi \cdot \overline{i \alpha \cdot \hat{x}\left(1-\frac{a}{m} \beta\right)(1+2 \mathbf{S} \cdot L) \psi} d x \\
& +\int_{\mathbb{R}^{3}}|x|\left|\left(1-\frac{a}{m} \beta\right)(1+2 \mathbf{S} \cdot L) \frac{\psi}{|x|}\right|^{2} d x=-\frac{m^{2}-a^{2}}{m^{2}} \int_{\mathbb{R}^{3}} \frac{|(1+2 \mathbf{S} \cdot L) \psi|^{2}}{|x|} d x .
\end{aligned}
$$

Let us firstly prove that

$$
\begin{array}{r}
-2 \operatorname{Re} \int_{\mathbb{R}^{3}}(-i \alpha \cdot \nabla+m \beta-a) \psi \cdot \overline{i \alpha \cdot \hat{x}\left(1-\frac{a}{m} \beta\right)(1+2 \mathbf{S} \cdot L) \psi} d x \\
=-2 \operatorname{Re} \int_{\mathbb{R}^{3}}(1+2 \mathbf{S} \cdot L) \frac{\psi}{|x|} \cdot \overline{\left(1-\frac{a}{m} \beta\right)(1+2 \mathbf{S} \cdot L) \psi} d x .
\end{array}
$$

With an explicit computation (see for example [25, Equation 4.102]) we get that

$$
-i \alpha \cdot \nabla=-i \alpha \cdot \hat{x}\left(\left(\partial_{r}+\frac{1}{|x|}\right) \mathbb{I}_{4}-\frac{1}{|x|}(1+2 \mathbf{S} \cdot L)\right),
$$

and so the last term in (2.2) can be expanded as follows:

$$
\begin{aligned}
-2 \operatorname{Re} \int_{\mathbb{R}^{3}}(-i \alpha & \cdot \nabla+m \beta-a) \psi \cdot \overline{i \alpha \cdot \hat{x}\left(1-\frac{a}{m} \beta\right)(1+2 \mathbf{S} \cdot L) \psi} d x \\
= & 2 \operatorname{Re} \int_{\mathbb{R}^{3}}\left(\partial_{r}+\frac{1}{|x|}\right) \psi \cdot \overline{\left(1-\frac{a}{m} \beta\right)(1+2 \mathbf{S} \cdot L) \psi} d x \\
& -2 \operatorname{Re} \int_{\mathbb{R}^{3}}(1+2 \mathbf{S} \cdot L) \frac{\psi}{|x|} \cdot \frac{\left(1-\frac{a}{m} \beta\right)(1+2 \mathbf{S} \cdot L) \psi}{\left(1 \alpha \cdot \hat{x}\left(1-\frac{a}{m} \beta\right)(1+2 \mathbf{S} \cdot L) \psi\right.} d x \\
& -2 \operatorname{Re} \int_{\mathbb{R}^{3}}(m \beta-a) \psi \cdot i I . \\
= & I+I I+I I I .
\end{aligned}
$$

We show that $I=I I I=0$. 
Indeed, the operator $\left(\partial_{r}+\frac{1}{|x|}\right)$ is skew-symmetric, and the operator $\left(1+\frac{a}{m} \beta\right)(1+2 \mathbf{S} \cdot L)$ is symmetric, since $\beta$ and $\mathbf{S} \cdot L$ are symmetric operators and they commute. Moreover,

$$
\left[\partial_{r}+\frac{1}{|x|},\left(1+\frac{a}{m} \beta\right)(1+2 \mathbf{S} \cdot L)\right]=0 .
$$

So we can conclude that $I=0$, thanks to Lemma 2.1.

Let us focus on III. Since $\beta^{2}=\mathbb{I}_{4}$, and $-i \alpha \cdot \hat{x}$ and $\beta$ anti-commute, we rewrite

$$
\begin{aligned}
I I I & =-2 \operatorname{Re} \int_{\mathbb{R}^{3}}(m \beta-a) \psi \cdot \overline{i \alpha \cdot \hat{x}\left(1-\frac{a}{m} \beta\right)(1+2 \mathbf{S} \cdot L) \psi} d x \\
& =\frac{1}{m} 2 \operatorname{Re} \int_{\mathbb{R}^{3}}(m \beta-a) \psi \cdot \overline{(m \beta+a) i \alpha \cdot \hat{x} \beta(1+2 \mathbf{S} \cdot L) \psi} d x \\
& =\frac{m^{2}-a^{2}}{m} 2 \operatorname{Re} \int_{\mathbb{R}^{3}} \psi \cdot \overline{i \alpha \cdot \hat{x} \beta(1+2 \mathbf{S} \cdot L) \psi} d x,
\end{aligned}
$$

where, in the last equality, we used the fact that $(m \beta+a)$ is symmetric. Since the operator $\beta(1+2 \mathbf{S} \cdot L)$ is symmetric, the operator $i \alpha \cdot \hat{x}$ is skew-symmetric and they anti-commute (see [25, Equation 4.108]), we have that the operator $i \alpha \cdot \hat{x} \beta(1+2 \mathbf{S} \cdot L)$ is skew-symmetric. Finally, the identity operator is symmetric, and it trivially commutes with $i \alpha \cdot \hat{x} \beta(1+2 \mathbf{S} \cdot L)$. Thus, Lemma 2.1 let us conclude that $I I I=0$, and so (2.4) is proved.

Finally, thanks to (2.4) we get that

$$
\begin{aligned}
\int_{\mathbb{R}^{3}}|x| \mid & \left.\left(1-\frac{a}{m} \beta\right)(1+2 \mathbf{S} \cdot L) \frac{\psi}{|x|}\right|^{2} d x-2 \operatorname{Re} \int_{\mathbb{R}^{3}}(1+2 \mathbf{S} \cdot L) \frac{\psi}{|x|} \cdot \overline{\left(1-\frac{a}{m} \beta\right)(1+2 \mathbf{S} \cdot L) \psi} d x \\
& =-\operatorname{Re} \int_{\mathbb{R}^{3}}\left(1+\frac{a}{m} \beta\right)(1+2 \mathbf{S} \cdot L) \psi \cdot \overline{\left(1-\frac{a}{m} \beta\right)(1+2 \mathbf{S} \cdot L) \frac{\psi}{|x|}} d x \\
& =-\frac{m^{2}-a^{2}}{m^{2}} \int_{\mathbb{R}^{3}} \frac{|(1+2 \mathbf{S} \cdot L) \psi|^{2}}{|x|} d x .
\end{aligned}
$$

and so (2.3) is proved. Finally we get (1.13) combining (2.1) and (A.5) .

Let us assume now that $\psi$ is a distribution verifying (1.12). Then, there exists a sequence $\left\{\varphi_{n}\right\}_{n} \subset C_{c}^{\infty}\left(\mathbb{R}^{3}\right)^{4}$ such that

$$
\varphi_{n} \rightarrow\left(H_{0}-a\right) \psi \quad \text { in } L^{2}(|x|)^{4} .
$$

The fundamental solution of $\left(H_{0}-a\right)$ is given by

$$
\phi^{a}(x):=\frac{e^{-\sqrt{m^{2}-a^{2}}|x|}}{4 \pi|x|}\left(a+m \beta+\left(1+\sqrt{m^{2}-a^{2}}|x|\right) i \alpha \cdot \frac{x}{|x|^{2}}\right) \quad \text { for } x \in \mathbb{R}^{3} \backslash\{0\},
$$

Since $\phi^{a}$ has exponential decay at infinity, we get that $\psi_{n}:=\phi^{a} * \varphi_{n} \in \mathcal{S}\left(\mathbb{R}^{3}\right)^{4}$, so it verifies

$$
\int_{\mathbb{R}^{3}}\left|\left(H_{0}-a\right) \psi_{n}\right|^{2}|x| d x \geq \frac{m^{2}-a^{2}}{m^{2}} \int_{\mathbb{R}^{3}} \frac{\left|(1+2 \mathbf{S} \cdot L) \psi_{n}\right|^{2}}{|x|} d x \geq \frac{m^{2}-a^{2}}{m^{2}} \int_{\mathbb{R}^{3}} \frac{\left|\psi_{n}\right|^{2}}{|x|} d x .
$$

By definition, $\left(H_{0}-a\right) \psi_{n}=\varphi_{n}$ and so we have that

$$
\left(H_{0}-a\right) \psi_{n} \rightarrow\left(H_{0}-a\right) \psi \quad \text { in } L^{2}(|x|)^{4} .
$$


Combining (2.7) and (2.6), we deduce that both $\left\{(1+2 \mathbf{S} \cdot L) \psi_{n}\right\}_{n}$ and $\left\{\psi_{n}\right\}_{n}$ are Cauchy sequences of $L^{2}\left(|x|^{-1}\right)^{4}$. So, there exist $\eta, \theta \in L^{2}\left(|x|^{-1}\right)^{4}$ such that

$$
\begin{gathered}
\psi_{n} \rightarrow \eta \quad \text { in } L^{2}\left(|x|^{-1}\right)^{4}, \\
(1+2 \mathbf{S} \cdot L) \psi_{n} \rightarrow \theta \quad \text { in } L^{2}\left(|x|^{-1}\right)^{4} .
\end{gathered}
$$

Taking the limit on $n$ in (2.6), we have that

$$
\int_{\mathbb{R}^{3}}\left|\left(H_{0}-a\right) \psi\right|^{2}|x| d x \geq \frac{m^{2}-a^{2}}{m^{2}} \int_{\mathbb{R}^{3}} \frac{|\theta|^{2}}{|x|} d x \geq \frac{m^{2}-a^{2}}{m^{2}} \int_{\mathbb{R}^{3}} \frac{|\eta|^{2}}{|x|} d x .
$$

Thanks to (2.8) we deduce that, in the sense of distributions, the following hold

$$
\begin{aligned}
\left(H_{0}-a\right) \psi_{n} & \rightarrow\left(H_{0}-a\right) \eta, \\
(1+2 \mathbf{S} \cdot L) \psi_{n} & \rightarrow(1+2 \mathbf{S} \cdot L) \eta .
\end{aligned}
$$

Thus, combining (2.7) with (2.11) and (2.9) with (2.12), we have that

$$
\begin{gathered}
\left(H_{0}-a\right) \psi=\left(H_{0}-a\right) \eta, \\
(1+2 \mathbf{S} \cdot L) \eta=\theta .
\end{gathered}
$$

Let us denote with $\langle\cdot, \cdot\rangle_{\mathcal{D}^{\prime}, \mathcal{D}}$ the usual pairing between a distribution and a test function. Then, for any $\varphi \in C_{c}^{\infty}\left(\mathbb{R}^{3}\right)^{4}$ we have that

$$
\langle\psi, \varphi\rangle_{\mathcal{D}^{\prime}, \mathcal{D}}=\int_{\mathbb{R}^{3}}\left(H_{0}-a\right) \psi \cdot \overline{\phi^{a} * \varphi} d x=\int_{\mathbb{R}^{3}}\left(H_{0}-a\right) \eta \cdot \overline{\phi^{a} * \varphi} d x=\langle\eta, \varphi\rangle_{\mathcal{D}^{\prime}, \mathcal{D}},
$$

where we used (2.13) in the third equality. For this reason, we can conclude that

$$
\psi=\eta \in L^{2}\left(|x|^{-1}\right)^{4}
$$

and thanks to $(2.14)$

$$
(1+2 \mathbf{S} \cdot L) \psi=(1+2 \mathbf{S} \cdot L) \eta=\theta .
$$

Finally, combining (2.10), (2.15), and (2.16) we can conclude that $\psi$ verifies (1.13). In particular, by a density argument, we get that $\psi$ verifies (2.1).

Let us finally assume that $\psi$ is an attainer of (1.13), that is

$$
\int_{\mathbb{R}^{3}}|(-i \alpha \cdot \nabla+m \beta-a) \psi|^{2}|x| d x=\frac{m^{2}-a^{2}}{m^{2}} \int_{\mathbb{R}^{3}} \frac{|(1+2 \mathbf{S} \cdot L) \psi|^{2}}{|x|} d x=\frac{m^{2}-a^{2}}{m^{2}} \int_{\mathbb{R}^{3}} \frac{|\psi|^{2}}{|x|} d x .
$$

We can decompose $\psi$ as in (A.3), that is

$$
\psi(x)=\sum_{j, k_{j}, m_{j}} \frac{1}{r}\left(f_{m_{j}, k_{j}}^{+}(r) \Phi_{m_{j}, k_{j}}^{+}(\hat{x})+f_{m_{j}, k_{j}}^{-}(r) \Phi_{m_{j}, k_{j}}^{-}(\hat{x})\right) .
$$

From the second equality of (2.17), and thanks to (A.4), we directly have $f_{m_{j}, k_{j}}^{ \pm}=0$ for $k_{j} \neq \pm 1$, or equivalently for $j \neq 1 / 2$. Let us focus on the first equality of (2.17). Thanks to (2.1), we get that

$$
0=\int_{\mathbb{R}^{3}}|x|\left|(-i \alpha \cdot \nabla+m \beta-a) \psi-i \alpha \cdot \hat{x}\left(1-\frac{a}{m} \beta\right)(1+2 \mathbf{S} \cdot L) \frac{\psi}{|x|}\right|^{2} d x
$$

and so,

$$
(-i \alpha \cdot \nabla+m \beta-a) \psi-i \alpha \cdot \hat{x}\left(1-\frac{a}{m} \beta\right)(1+2 \mathbf{S} \cdot L) \frac{\psi}{|x|}=0
$$


Multiplying both therms by $i \alpha \cdot \hat{x}$ and using (2.5) we get that

$$
\left(\partial_{r}+\frac{1}{|x|}+i \alpha \cdot \hat{x}(m \beta-a)\right) \psi-\frac{a}{m} \beta(1+2 \mathbf{S} \cdot L) \frac{\psi}{|x|}=0 .
$$

The action of all the operators appearing in (2.18) leaves invariant the decomposition in partial wave subspaces. Thanks to (A.1), we get that for $m_{1 / 2}= \pm 1 / 2$ and $k_{1 / 2}= \pm 1$ we have

$$
\left(\begin{array}{cc}
\partial_{r}+\frac{a k_{1 / 2}}{m r} & -(m+a) \\
-(m-a) & \partial_{r}+\frac{a k_{1 / 2}}{m r}
\end{array}\right) \cdot\left(\begin{array}{l}
f_{m_{1 / 2}, k_{1 / 2}}^{+} \\
f_{m_{1 / 2}, k_{1 / 2}}^{-}
\end{array}\right)=0 .
$$

The only solution of (2.19) that is integrable at $+\infty$ is

$$
\left(\begin{array}{l}
f_{m_{1 / 2}, k_{1 / 2}}^{+} \\
f_{m_{1 / 2}, k_{1 / 2}}^{-}
\end{array}\right)=\left(\begin{array}{c}
e^{-\sqrt{m^{2}-a^{2}} r} r^{-a k_{1 / 2} / m} \\
-\sqrt{\frac{m+a}{m-a}} e^{-\sqrt{m^{2}-a^{2}} r} r^{-a k_{1 / 2} / m}
\end{array}\right) .
$$

Then $\left(f_{m_{1 / 2}, k_{1 / 2}}^{+}, f_{m_{1 / 2}, k_{1 / 2}}^{-}\right) \in L^{2}(0,+\infty)^{2}$ if and only if $a k_{1 / 2} \leq 0$. So if $a>0$,we have to assume $k_{1 / 2}=-1$, and if $a<0$ we have $k_{1 / 2}=1$. Remembering that

$$
\begin{aligned}
& \Phi_{\frac{1}{2},-1}^{+}=\frac{1}{\sqrt{4 \pi}}\left(\begin{array}{l}
i \\
0 \\
0 \\
0
\end{array}\right), \\
& \Phi_{-\frac{1}{2},-1}^{+}=\frac{1}{\sqrt{4 \pi}}\left(\begin{array}{l}
0 \\
i \\
0 \\
0
\end{array}\right), \\
& \Phi_{\frac{1}{2},-1}^{-}=\frac{1}{\sqrt{4 \pi}}\left(\begin{array}{c}
0 \\
0 \\
\sigma \cdot \hat{x} \cdot\left(\begin{array}{l}
0 \\
1
\end{array}\right)
\end{array}\right), \\
& \Phi_{-\frac{1}{2},-1}^{-}=\frac{1}{\sqrt{4 \pi}}\left(\begin{array}{c}
0 \\
0 \\
\sigma \cdot \hat{x} \cdot\left(\begin{array}{l}
0 \\
1
\end{array}\right)
\end{array}\right) \text {, }
\end{aligned}
$$

we conclude the proof.

\section{Birman-Schwinger Principle for the DiraC-Coulomb operator}

This section is devoted to the proof of Theorem 1.3.

Let $a \in(-m, m), a \in \sigma_{d}\left(H_{D}\right)$ and $\psi \in \mathcal{D}\left(H_{D}\right) \backslash\{0\}$ such that $\left(H_{0}+\mathbf{V}-a\right) \psi=0$. We have that, in the sense of distributions,

$$
\left(H_{0}-a\right) \psi=-\mathbf{V} \psi .
$$

Since $\psi \in \mathcal{D}\left(H_{D}\right) \subset \mathcal{D}\left(r^{-1 / 2}\right)^{4}$, then $f:=\mathbf{u} \psi \in L^{2}\left(\mathbb{R}^{3}\right)^{4}$ and $\mathbf{v} f \in \mathcal{D}\left(r^{1 / 2}\right)^{4}$. Thanks to (1.15) we can apply $\left(H_{0}-a\right)^{-1}$ to $(3.1)$, getting $\psi=-\left(H_{0}-a\right)^{-1} \mathbf{v} f$, that implies

$$
f=\mathbf{u} \psi=-\mathbf{u}\left(H_{0}-a\right)^{-1} \mathbf{v} f .
$$

Let now -1 be an eigenvalue of $\mathbf{u}\left(H_{0}-a\right)^{-1} \mathbf{v}$ and let $f \in L^{2}\left(\mathbb{R}^{3}\right)^{4}$ be an eigenfunction. Setting $\psi=\left(H_{0}-a\right)^{-1} \mathbf{v} f$, we directly get that $\psi \in \mathcal{D}\left(r^{-1 / 2}\right)$. Reasoning as above, we get that $H_{D} \psi=a \psi$, and so $\psi \in H_{D}$ and $\psi$ is an eigenfunction of the eigenvalue $a$.

Finally, we point out that the shown procedure ensures that the multiplicity of $a$ as an eigenvalue of $H_{D}$ coincides with the multiplicity of -1 as an eigenvalue of $\mathbf{u}\left(H_{0}-a\right)^{-1} \mathbf{v}$, and this concludes the proof. 


\section{Proof of Theorem 1.4}

This section is devoted to the proofs of Theorem 1.4 and Corollary 1.6.

Proof of Theorem 1.4. Let $a \in(-m, m)$ and assume that there exists $\psi \in \mathcal{D}\left(H_{D}\right) \backslash\{0\}$ such that $\left(H_{D}-a\right) \psi=0$. Then, in the sense of distributions, we get that $\left(H_{0}-a\right) \psi=-\mathbf{V} \psi$. Since $\psi \in \mathcal{D}\left(H_{D}\right) \subset \mathcal{D}\left(r^{-1 / 2}\right)^{4}$ and thanks to the fact that $\mathbf{V}$ verifies (1.5), we get that

$$
\int_{\mathbb{R}^{3}}|x|\left|\left(H_{0}-a\right) \psi\right|^{2} d x=\int_{\mathbb{R}^{3}}|x||\mathbf{V} \psi|^{2} d x \leq \nu^{2} \int_{\mathbb{R}^{3}} \frac{|\psi|^{2}}{|x|} d x<+\infty .
$$

Thanks to Theorem 1.1 we get that $\psi$ verifies (1.13), and so

$$
\begin{aligned}
\nu^{2} \int_{\mathbb{R}^{3}} \frac{|\psi|^{2}}{|x|} & \geq \int_{\mathbb{R}^{3}}|x|\left|\left(H_{0}-a\right) \psi\right|^{2} d x \geq \frac{m^{2}-a^{2}}{m^{2}} \int_{\mathbb{R}^{3}} \frac{|(1+2 \mathbf{S} \cdot L) \psi|^{2}}{|x|} d x \\
& \geq \frac{m^{2}-a^{2}}{m^{2}} \int_{\mathbb{R}^{3}} \frac{|\psi|^{2}}{|x|} d x,
\end{aligned}
$$

So, $\nu^{2} \geq \frac{m^{2}-a^{2}}{m^{2}}$, that directly implies (i).

Let us prove (ii). Let us assume that $a^{2}=m^{2}\left(1-\nu^{2}\right)$. Then, from (4.1) we deduce that $\psi$ is an attainer of (1.13): thanks to Theorem 1.1, this is equivalent to say that there exists $C \in \mathbb{C}^{2}$ such that $\psi=\psi_{C}^{a}$, with $\psi_{C}^{a}$ defined in (1.14). This directly implies that $\mu(a) \leq 2$. Finally, thanks to (1.18) and (1.19) we get that $0=\left(H_{0}-\operatorname{sign}(a) \frac{\nu}{|x|}-a\right) \psi_{C}^{a}=\left(H_{0}+\mathbf{V}-a\right) \psi_{C}^{a}$, so $\mathbf{V} \psi_{C}^{a}=-\operatorname{sign}(a) \frac{\nu}{|x|} \psi_{C}^{a}$.

Let us now prove (iii). We assume that $a$ is positive, that is $a=m \sqrt{1-\nu^{2}}$, since the same approach can be used when $a$ is negative. Moreover, let us assume that, for any $C \in \mathbb{C}^{2}$,

$$
\mathbf{V} \psi_{C}^{a}=-\frac{\nu}{|x|} \psi_{C}^{a} \text {. }
$$

Since

$$
\sqrt{\frac{m-a}{m+a}}=\sqrt{\frac{1-\sqrt{1-\nu^{2}}}{1+\sqrt{1-\nu^{2}}}}=\mathrm{N}
$$

we have that

$$
\psi_{C}^{a}=\frac{e^{-\sqrt{m^{2}-a^{2}}|x|}}{|x|^{1-a / m}}\left(\begin{array}{cc}
\mathbb{I}_{2} & 0 \\
0 & i \mathrm{~N} \sigma \cdot \hat{x}
\end{array}\right) \cdot\left(\begin{array}{l}
C \\
C
\end{array}\right),
$$

where, with abuse of notation, we are denoting with $\left(\begin{array}{l}C \\ C\end{array}\right)$ the 4 -component column vector.

Thanks to (4.4), multiplying both therms of (4.2) by $\left(\frac{e^{-\sqrt{m^{2}-a^{2}}|x|}}{|x|^{1-a / m}}\right)^{-1}$, we get that

$$
\left(\mathbf{V}(x)+\frac{\nu}{|x|} \mathbb{I}_{4}\right) \cdot\left(\begin{array}{cc}
\mathbb{I}_{2} & 0 \\
0 & i \mathrm{~N} \sigma \cdot \hat{x}
\end{array}\right) \cdot\left(\begin{array}{l}
C \\
C
\end{array}\right)=0 .
$$

Since both $\mathbf{V}$ and $\frac{\nu}{|x|} \mathbb{I}_{4}$ are Hermitian matrices, we can write

$$
\left(\mathbf{V}+\frac{\nu}{|x|} \mathbb{I}_{4}\right)=:\left(\begin{array}{ll}
\mathbf{W}_{1,1} & \mathbf{W}_{1,2} \\
\mathbf{W}_{1,2}^{*} & \mathbf{W}_{2,2}
\end{array}\right),
$$


where $\mathbf{W}_{1,1}$ and $\mathbf{W}_{2,2}$ are $2 \times 2$ Hermitian matrices, $\mathbf{W}_{1,2}$ is a $2 \times 2$ complex valued matrix and $\mathbf{W}_{1,2}^{*}$ is its adjoint matrix.

Combining (4.5) and (4.6), we get that

$$
\left\{\begin{array}{l}
\left(\mathbf{W}_{1,1}+i \mathrm{NW}_{1,2} \sigma \cdot \hat{x}\right) \cdot C=0, \\
\left(\mathbf{W}_{1,2}^{*}+i \mathbf{N W}_{2,2} \sigma \cdot \hat{x}\right) \cdot C=0 .
\end{array}\right.
$$

Since (4.7) holds for any $C \in \mathbb{C}^{2}$, we deduce that

$$
\begin{aligned}
& \mathbf{W}_{1,1}+i \mathbf{N W}_{1,2} \sigma \cdot \hat{x}=0, \\
& \mathbf{W}_{1,2}^{*}+i \mathbf{N W}_{2,2} \sigma \cdot \hat{x}=0 .
\end{aligned}
$$

Taking the adjoint of (4.9), and thanks to the fact that both $\sigma \cdot \hat{x}$ and $\mathbf{W}_{2,2}$ are Hermitian matrices, we get that

$$
\mathbf{W}_{1,2}=i \mathrm{~N} \sigma \cdot \hat{x} \mathbf{W}_{2,2} .
$$

Combining (4.8) and (4.10) we get that

$$
\mathbf{W}_{1,1}=\mathrm{N}^{2} \sigma \cdot \hat{x} \mathbf{W}_{2,2} \sigma \cdot \hat{x} .
$$

Setting for convenience $\mathbf{W}^{+}:=\mathbf{W}_{2,2}$, we can conclude that (4.5) is equivalent to

$$
\mathbf{V}(x):=-\frac{\nu}{|x|} \mathbb{I}_{4}+\left(\begin{array}{cc}
\mathrm{N}^{2} \sigma \cdot \hat{x} \mathbf{W}^{+}(x) \sigma \cdot \hat{x} & i \mathrm{~N} \sigma \cdot \hat{x} \mathbf{W}^{+}(x) \\
-i \mathbf{N W}^{+}(x) \sigma \cdot \hat{x} & \mathbf{W}^{+}(x)
\end{array}\right) .
$$

Finally, thanks to (1.5) we determine additional properties on the matrix $\mathbf{W}^{+}(x)$. For any $x \in \mathbb{R}^{3} \backslash\{0\}$, there exists $\left\{e_{1}(x), e_{2}(x)\right\}$, an orthonormal basis of $\mathbb{C}^{2}$ of eigenvectors of $\mathbf{W}^{+}(x)$, that is $\mathbf{W}^{+}(x) e_{j}(x)=\lambda_{j}^{+}(x) e_{j}(x)$, for $j=1,2$, with $\lambda_{j}^{+}(x) \in \mathbb{R}$. Set

$$
u_{j}(x):=\frac{1}{N^{2}+1}\left(\begin{array}{c}
e_{j}(x) \\
i \mathrm{~N} \sigma \cdot \hat{x} e_{j}(x)
\end{array}\right) \quad \text { and } \quad v_{j}(x):=\frac{1}{N^{2}+1}\left(\begin{array}{c}
i \mathrm{~N} \sigma \cdot \hat{x} e_{j}(x) \\
e_{j}(x)
\end{array}\right), \quad \text { for } j=1,2 .
$$

The family $\left\{u_{1}(x), u_{2}(x), v_{1}(x), v_{2}(x)\right\}$ is an orthonormal basis of $\mathbb{C}^{4}$. Thus, $|x||\mathbf{V}(x)| \leq \nu$ if and only if $|x|\left|\mathbf{V}(x) u_{j}(x)\right| \leq \nu$ and $|x|\left|\mathbf{V}(x) v_{j}(x)\right| \leq \nu$ for $j=1,2$.

We have that, for $j=1,2$

$$
\begin{aligned}
& \mathbf{V}(x) u_{j}(x)=-\frac{\nu}{|x|} u_{j}(x), \\
& \mathbf{V}(x) v_{j}(x)=\left(-\frac{\nu}{|x|}+\lambda_{j}^{+}(x)\left(N^{2}+1\right)\right) v_{j}(x) .
\end{aligned}
$$

Since $\left|u_{j}(x)\right|=\left|v_{j}(x)\right|=1$, from (4.12) we deduce that that $|x||\mathbf{V}(x)| \leq \nu$ if and only if

$$
\left(-\nu+|x| \lambda_{j}^{+}(x)\left(N^{2}+1\right)\right)^{2} \leq \nu^{2}, \quad \text { for } j=1,2 .
$$

From (4.13) and (4.3) we deduce (1.21), concluding the proof.

Proof of Corollary 1.6. From (ii) in Theorem 1.4 we have that $V(x) \psi_{C}^{a}=\mp \frac{\nu}{|x|} \psi_{C}^{a}$ for some $C \in \mathbb{C}^{2}$, and this implies the thesis. 


\section{Appendix A. Partial wave subspaces}

In this appendix, we recall the partial wave subspaces associated to the Dirac equation. We sketch here this topic, referring to [25, Section 4.6] for further details.

Let $Y_{n}^{l}$ be the spherical harmonics. They are defined for $n=0,1,2, \ldots$, and $l=-n,-n+$ $1, \ldots, n$, and they satisfy $\Delta_{\mathbb{S}^{2}} Y_{n}^{l}=n(n+1) Y_{n}^{l}$, where $\Delta_{\mathbb{S}^{2}}$ denotes the usual spherical Laplacian. Moreover, $Y_{n}^{l}$ form a complete orthonormal set in $L^{2}\left(\mathbb{S}^{2}\right)$. For $j=1 / 2,3 / 2,5 / 2, \ldots$, and $m_{j}=$ $-j,-j+1, \ldots, j$, set

$$
\begin{aligned}
& \psi_{j-1 / 2}^{m_{j}}:=\frac{1}{\sqrt{2 j}}\left(\begin{array}{c}
\sqrt{j+m_{j}} Y_{j-1 / 2}^{m_{j}-1 / 2} \\
\sqrt{j-m_{j}} Y_{j-1 / 2}^{m_{j}+1 / 2}
\end{array}\right), \\
& \psi_{j+1 / 2}^{m_{j}}:=\frac{1}{\sqrt{2 j+2}}\left(\begin{array}{c}
\sqrt{j+1-m_{j}} Y_{j+1 / 2}^{m_{j}-1 / 2} \\
-\sqrt{j+1+m_{j}} Y_{j+1 / 2}^{m_{j}+1 / 2}
\end{array}\right) ;
\end{aligned}
$$

then $\psi_{j \pm 1 / 2}^{m_{j}}$ form a complete orthonormal set in $L^{2}\left(\mathbb{S}^{2}\right)^{2}$. For $k_{j}:= \pm(j+1 / 2)$ we set

$$
\Phi_{m_{j}, \pm(j+1 / 2)}^{+}:=\left(\begin{array}{c}
i \psi_{j \pm 1 / 2}^{m_{j}} \\
0
\end{array}\right), \quad \Phi_{m_{j}, \pm(j+1 / 2)}^{-}:=\left(\begin{array}{c}
0 \\
\psi_{j \neq 1 / 2}^{m_{j}}
\end{array}\right) .
$$

Then, the set $\left\{\Phi_{m_{j}, k_{j}}^{+}, \Phi_{m_{j}, k_{j}}^{-}\right\}_{j, k_{j}, m_{j}}$ is a complete orthonormal basis of $L^{2}\left(\mathbb{S}^{2}\right)^{4}$ and

$$
(1+2 \mathbf{S} \cdot L) \Phi_{m_{j}, k_{j}}=-k_{j} \beta \Phi_{m_{j}, k_{j}}
$$

where the spin angular momentum operator $\mathbf{S}$ and the orbital angular momentum $L$ are defined as

$$
\mathbf{S}=\frac{1}{2}\left(\begin{array}{cc}
\sigma & 0 \\
0 & \sigma
\end{array}\right) \quad \text { and } \quad L:=-i x \wedge \nabla
$$

So, we can write

$$
\psi(x)=\sum_{j, k_{j}, m_{j}} \frac{1}{r}\left(f_{m_{j}, k_{j}}^{+}(r) \Phi_{m_{j}, k_{j}}^{+}(\hat{x})+f_{m_{j}, k_{j}}^{-}(r) \Phi_{m_{j}, k_{j}}^{-}(\hat{x})\right)
$$

and, by definition,

$$
\int_{\mathbb{R}^{3}}|\psi|^{2} d x=\sum_{j, k_{j}, m_{j}} \int_{0}^{+\infty}\left|f_{m_{j}, k_{j}}^{+}(r)\right|^{2}+\left|f_{m_{j}, k_{j}}^{-}(r)\right|^{2} d r .
$$

Thanks to [25, Equation 4.109] and (A.3), we have that

$$
\begin{aligned}
\int_{\mathbb{R}^{3}} \frac{|\psi|^{2}}{|x|} d x & =\sum_{j, k_{j}, m_{j}} \int_{0}^{+\infty} \frac{1}{r}\left(\left|f_{m_{j}, k_{j}}^{+}(r)\right|^{2}+\left|f_{m_{j}, k_{j}}^{-}(r)\right|^{2}\right) d r \\
\int_{\mathbb{R}^{3}} \frac{|(1+2 \mathbf{S} \cdot L) \psi|^{2}}{|x|} d x & =\sum_{j, k_{j}, m_{j}} \int_{0}^{+\infty} \frac{k_{j}^{2}}{r}\left(\left|f_{m_{j}, k_{j}}^{+}(r)\right|^{2}+\left|f_{m_{j}, k_{j}}^{-}(r)\right|^{2}\right) d r .
\end{aligned}
$$

From (A.4), we directly deduce that

$$
\int_{\mathbb{R}^{3}} \frac{|\psi|^{2}}{|x|} d x \leq \int_{\mathbb{R}^{3}} \frac{|(1+2 \mathbf{S} \cdot L) \psi|^{2}}{|x|} d x,
$$

and that (A.5) is attained if and only if $f_{m_{j}, k_{j}}^{ \pm}=0$ for $k_{j} \neq \pm 1$, or equivalently $j \neq 1 / 2$. 


\section{REFERENCES}

[1] Arai, M. On essential selfadjointness, distinguished selfadjoint extension and essential spectrum of Dirac operators with matrix valued potentials. Publications of the Research Institute for Mathematical Sciences 19, 1 (1983), 33-57.

[2] Arrizabalaga, N., Duonndikoetxea, J., And Vega, L. Self-adjoint extensions of Dirac operators with Coulomb type singularity. Journal of Mathematical Physics 54, 4 (2013), 041504.

[3] Arrizabalaga, N., Duoandikoetxea, J., and Vega, L. Erratum: "Self-adjoint extensions of Dirac operators with Coulomb type singularity" [J. Math. Phys. 54, 041504 (2013)]. Journal of Mathematical Physics 59, 7 (2018), 079902.

[4] Burnap, C., Brysk, H., And Zweifel, P. Dirac Hamiltonian for strong Coulomb fields. Il Nuovo Cimento $B$ (1971-1996) 64, 2 (1981), 407-419.

[5] Cassano, B., And Pizzichillo, F. Self-adjointness for the Dirac operator with Coulomb-type spherically symmetric perturbations via boundary conditions. To appear (2018).

[6] Cassano, B., And Pizzichillo, F. Self-adjoint extensions for the Dirac operator with Coulomb-type spherically symmetric potentials. Letters in Mathematical Physics (2018), 1-33.

[7] Dolbeault, J., Esteban, M. J., Loss, M., And Vega, L. An analytical proof of Hardy-like inequalities related to the Dirac operator. Journal of Functional Analysis 216, 1 (2004), 1-21.

[8] Dolbeault, J., Esteban, M. J., And Séré, E. On the eigenvalues of operators with gaps. Application to Dirac operators. Journal of Functional Analysis 174, 1 (2000), 208-226.

[9] Esteban, M. J., Lewin, M., And SÉRÉ, E. Domains for Dirac-Coulomb min-max levels. arXiv preprint arXiv:1702.04976 (2017).

[10] Esteban, M. J., And Loss, M. Self-adjointness for Dirac operators via Hardy-Dirac inequalities. Journal of Mathematical Physics 48, 11 (2007), 112107.

[11] Gallone, M., and Michelangeli, A. Discrete spectra for critical Dirac-Coulomb Hamiltonians. arXiv preprint arXiv:1710.11389 (2017).

[12] Gallone, M., And Michelangeli, A. Self-adjoint realisations of the Dirac-Coulomb Hamiltonian for heavy nuclei. arXiv preprint arXiv:1706.00700 (2017).

[13] Gustafson, K., and Rejto, P. Some essentially self-adjoint Dirac operators with spherically symmetric potentials. Israel Journal of Mathematics 14, 1 (1973), 63-75.

[14] Hardy, G. H. Note on a theorem of Hilbert. Mathematische Zeitschrift 6, 3-4 (1920), 314-317.

[15] Hogreve, H. The overcritical Dirac-Coulomb operator. Journal of Physics A: Mathematical and Theoretical 46, 2 (2012), 025301.

[16] Kato, T. Fundamental properties of Hamiltonian operators of Schrödinger type. Transactions of the American Mathematical Society 70, 2 (1951), 195-211.

[17] Kato, T. Holomorphic families of Dirac operators. Mathematische Zeitschrift 183, 3 (1983), 399-406.

[18] Kato, T. Perturbation theory for linear operators, vol. 132. Springer Science \& Business Media, 2013.

[19] Klaus, M., And Wüst, R. Characterization and uniqueness of distinguished self-adjoint extensions of Dirac operators. Communications in Mathematical Physics 64, 2 (1979), 171-176.

[20] Kufner, A., Maligranda, L., And Persson, L.-E. The prehistory of the Hardy inequality. The American Mathematical Monthly 113, 8 (2006), 715-732.

[21] Nenciu, G. Self-adjointness and invariance of the essential spectrum for Dirac operators defined as quadratic forms. Communications in Mathematical Physics 48, 3 (1976), 235-247.

[22] Rellich, F., And Jörgens, K. Eigenwerttheorie partieller Differentialgleichungen: Vorlesung, gehalten an der Universität Göttingen. Mathematisches Institut der Universität Göttingen, 1953.

[23] Schmincke, U.-W. Distinguished selfadjoint extensions of Dirac operators. Mathematische Zeitschrift 129, 4 (1972), 335-349.

[24] Schmincke, U.-W. Essential selfadjointness of Dirac operators with a strongly singular potential. Mathematische Zeitschrift 126, 1 (1972), 71-81.

[25] Thaller, B. The Dirac equation, vol. 31. Springer-Verlag Berlin, 1992.

[26] Voronov, B. L., Gitman, D. M., and Tyutin, I. V. The Dirac Hamiltonian with a superstrong Coulomb field. Theoretical and Mathematical Physics 150, 1 (2007), 34-72.

[27] Weidmann, J. Oszillationsmethoden für systeme gewöhnlicher Differentialgleichungen. Mathematische Zeitschrift 119, 4 (1971), 349-373.

[28] WÜst, R. Distinguished self-adjoint extensions of Dirac operators constructed by means of cut-off potentials. Mathematische Zeitschrift 141, 1 (1975), 93-98. 
[29] XIA, J. On the contribution of the Coulomb singularity of arbitrary charge to the Dirac Hamiltonian. Transactions of the American Mathematical Society 351, 5 (1999), 1989-2023.

B. Cassano, Department of Theoretical Physics, NPI, Academy of Sciences, 25068 Řež (Czechia) E-mail address: cassano@ujf.cas.cz

F. Pizzichillo, BCAM - Basque Center for Applied Mathematics, Alameda de Mazarredo 14, 48009 Bilbao (Spain)

E-mail address: fpizzichillo@bcamath.org

L. Vega, BCAM - Basque Center for Applied Mathematics, Alameda de Mazarredo 14, 48009 Bilbao (SPAin)

E-mail address: lvega@bcamath.org 